\title{
Spinal epidural lipomatosis: a review of its causes and recommendations for treatment
}

\author{
Daniel R. Fassett, M.D., M.B.A., ANd Meic H. Schmidt, M.D. \\ Department of Neurosurgery, University of Utah School of Medicine, Salt Lake City, Utah
}

\begin{abstract}
Spinal epidural lipomatosis is most commonly observed in patients receiving long-term exogenous steroid therapy, but can also be seen in patients with endogenous steroid overproduction, obesity, or idiopathic disease. With this condition, there is hypertrophy of the epidural adipose tissue, causing a narrowing of the spinal canal and compression of neural structures. A majority of patients will present with progressive myelopathy, but radicular symptoms are also common. Conservative treatment-weaning from steroids or weight loss — can reverse the hypertrophy of the adipose tissue and relieve the neural compression. If conservative management fails, surgery with decompressive laminectomy is also very successful at improving the patient's neurological symptoms.
\end{abstract}

KEY WoRdS • lipomatosis • spinal • steroid

Spinal epidural lipomatosis is a rare and complex disorder that can present with progressive neurological deficits. Hypertrophy of adipose tissue located in the spinal epidural space is most commonly associated with long-term steroid use but can be observed in patients with a number of other conditions. As the adipose tissue enlarges, it encroaches on the spinal canal and compresses the neural elements. We have reviewed the literature on SEL and summarize current trends in the management of this condition.

\section{CAUSES OF SEL}

Most reported cases of SEL are associated with longterm use of exogenous steroids, but a number of non-steroid-related cases have been reported, including some associated with Cushing disease, Cushing syndrome, hypothyroidism, ${ }^{17}$ pituitary prolactinoma, ${ }^{5}$ and obesity. ${ }^{2,8,13}$ A number of idiopathic cases have also been reported. ${ }^{1,3,9,11,18}$ The disorder is thought to be part of the spectrum of abnormal adipose deposition that is observed in patients with Cushing syndrome. It appears to occur more commonly in men than women, with $75 \%$ of reported cases involving male patients. ${ }^{13,14,18}$ The mean age of patients with SEL who have been described in the literature is 43 years, but the disorder tends to occur at a younger age in male patients. ${ }^{13,14,18}$ The youngest reported case was in a 6-year-old boy who was receiving high-dose steroid therapy. ${ }^{12}$

Among the series that have been reported, approximately $75 \%$ of cases have been associated with exogenous steroid use. ${ }^{18}$ Dosages and duration of steroid use vary

Abbreviations used in this paper: $\mathrm{CT}=$ computerized tomography; MR = magnetic resonance; SEL = spinal epidural lipomatosis. widely in patients in whom this condition develops. In most cases, moderate-to-high steroid dosages have been received for years before the development of symptoms. The mean daily doses of prednisone in SEL series in the literature have ranged from 30 to $100 \mathrm{mg}$ and the mean duration of steroid use before the development of symptoms have ranged from 5 to 11 years. ${ }^{4}$ Nevertheless, this condition has developed in some patients who have used steroids for months and at relatively low dosages., ${ }^{41}$ There have been reports of SEL associated with epidural steroid injections and even inhaled steroids, but long-term use of oral steroids is by far the most common association. ${ }^{10,14,15}$

\section{FINDINGS OF SEL}

\section{Clinical Findings}

Back pain is the most frequently reported symptom associated with SEL and often presents long before the other symptoms. Lower-extremity weakness is also a common complaint and appears to be slowly progressive in most cases. Sensory changes with numbness, paresthesias, or radicular symptoms are also common. Bowel and bladder incontinence are reported, but appear to be rare complaints. On physical examination, lower-extremity weakness is the most common finding with decreased pinprick sensation and altered reflexes also frequently occurring. $4,13,14$

Obviously, the symptoms are somewhat dependent on the level of canal compromise (spinal cord, conus medullaris, or cauda equina), with thoracic levels producing myelopathic effects and lumbar levels having radicular effects. A majority of these patients present with thoracic epidural lipomatosis and, therefore, progressive myelopathy is a more common presentation than radicular complaints. 


\section{Imaging Findings}

Although SEL cannot be diagnosed on plain x-ray films, these studies can help rule out more sinister causes of back pain such as degenerative disease and tumors. During the pre-MR imaging era, diagnosis was based on findings on myelograms and CT scans. Myelography reveals an obstruction to the contrast dye at the level of the canal compression, and the filling pattern is helpful in determining whether the obstruction is intradural or extradural. An hourglass pattern of contrast obstruction is usually associated with an epidural mass such as SEL. Axial CT scans are used to help differentiate the cause of the compression based on the density of the tissue in the epidural space. Adipose tissue has a density ranging between -80 and $-120 \mathrm{HU}$ on CT scans; this differentiates it from most other tissues in the area. ${ }^{14,16}$

Magnetic resonance imaging has replaced CT scanning as the study of choice for the diagnosis of SEL. Canal compression with obliteration of the cerebrospinal fluid spaces can be appreciated on axial and sagittal MR images, and a high-signal intensity on $\mathrm{T}_{1}$-weighted images and an intermediate signal on $\mathrm{T}_{2}$-weighted images are characteristic of adipose tissue. Epidural adipose tissue that has a thickness greater than $7 \mathrm{~mm}$ has been reported to be the diagnostic criterion for SEL (Fig. 1). ${ }^{8,13}$ Spinal epidural lipomatosis should be differentiated from encapsulated spinal lipomas, which can also cause epidural spinal compression.

Thoracic involvement is most common, with between 58 and $61 \%$ of cases involving the thoracic cord. Lumbar involvement is found in 39 to $42 \%$ of cases and cervical SEL has never been reported. ${ }^{4,18}$ Some clinicians have

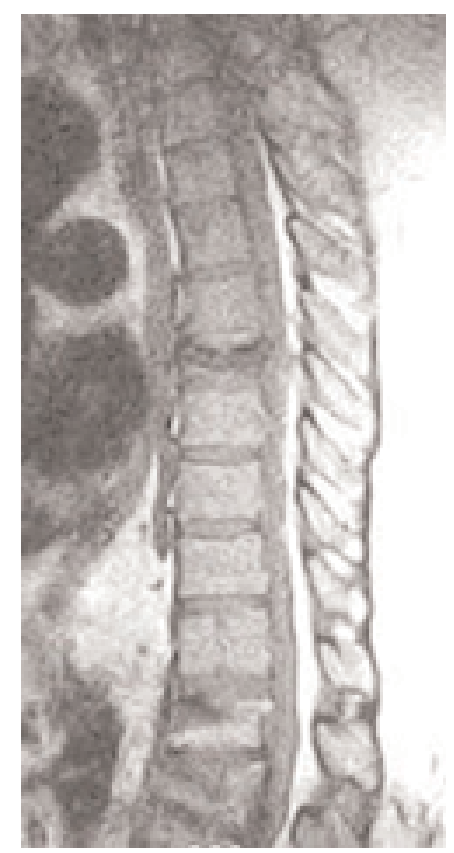

Fig. 1. Sagittal $\mathrm{T}_{1}$-weighted MR image of the thoracic spine of a 36-year-old man who engaged in long-term steroid usage. This image demonstrates hypertrophy of adipose tissue located posterior to T5-L2. Compression fractures are also noted. suggested that female patients are more likely to have lumbar SEL. ${ }^{14}$ Often there is an asymmetrical deposition of adipose tissue in the epidural space with greater adipose hypertrophy in the dorsal and lateral regions of this space. ${ }^{18}$

Although MR imaging is the study of choice for most cases, clinicians should familiarize themselves with the myelography and CT characteristics of SEL, because a portion of these patients will be unable to undergo MR imaging because of body habitus and other factors.

\section{TREATMENT OF SEL}

Treatment options include conservative therapy and surgical intervention. Given the limited number of cases, clinical trials have not been performed to compare outcomes of conservative and surgical management. Conservative therapy, including weaning of patients from steroids and weight loss, has been successful in a number of cases. ${ }^{2,4,13,18}$ Nevertheless, many patients receive steroid medications for other chronic illnesses and may not tolerate weaning from the therapy. Weight loss has been reported to be very successful in patients with SEL in whom obesity is thought to be the cause of the adipose hypertrophy. ${ }^{2,11,13}$ Patients not receiving steroids should undergo an endocrinological evaluation for possible endogenous corticosteroid overproduction. In most cases, treatment of endogenous corticosteroid overproduction should be undertaken before spinal decompression is considered.

Surgical intervention, with decompressive laminectomy and resection of epidural adipose tissue, has reportedly been successful in a large percentage of patients. It appears that a majority of these patients experience improvement or resolution of their neurological symptoms after surgical intervention. ${ }^{13}$ Patients with pathological conditions in the lumbar (cauda equina) region tend to make a better recovery following surgical decompression than patients with pathological conditions in the thoracic region. ${ }^{14}$ Preoperative steroid doses are also reported to influence neurological recovery, with patients receiving lower doses of steroids having a better chance for recovery. ${ }^{14}$ It also appears that patients with idiopathic SEL tend to have a better prognosis, with most attaining complete recovery after surgical decompression., ${ }^{7,11,13}$ Some patients have been known to require multiple surgeries because of reaccumulation of epidural fat, but this appears to be very rare. ${ }^{6}$

Although the surgical procedure itself is low risk, the postoperative management of these patients' concomitant medical problems and comorbidities can be fraught with complications and morbidity. Fessler and colleagues ${ }^{4}$ reported a $22 \%$ mortality rate in these patients within 1 year after surgical decompression. They recommend attempting conservative treatment for patients without significant cord compression. Given that this process is a slow gradual compression of the neural structures, there is no evidence to suggest that rapid decompression will result in improved recovery. We favor a more conservative strategy, especially in patients with significant comorbidities, and reserve surgical decompression for patients in whom conservative measures fail. 


\section{CONCLUSIONS}

Spinal epidural lipomatosis is most commonly associated with long-term use of exogenous steroids, but can be seen with a number of other conditions. Conservative treatment, with either weight loss or weaning from steroids, can successfully reduce epidural adipose tissue leading to an improvement in symptoms. Decompressive laminectomy with resection of epidural adipose tissue is also very successful at improving neurological symptoms. Given the significant comorbidities in many of these patients, we would recommend reserving surgical intervention for patients in whom conservative treatment fails.

\section{References}

1. Bednar DA, Esses SI, Kucharczyk W: Symptomatic lumbar epidural lipomatosis in a normal male. A unique case report. Spine 15:52-53, 1990

2. Beges C, Rousselin B, Chevrot A, et al: Epidural lipomatosis. Interest of magnetic resonance imaging in a weight-reduction treated case. Spine 19:251-254, 1994

3. Citow JS, Kranzler L: Thoracic epidural lipomatosis with associated syrinx: case report. Surg Neurol 53:589-591, 2000

4. Fessler RG, Johnson DL, Brown FD, et al: Epidural lipomatosis in steroid-treated patients. Spine 17:183-188, 1992

5. Fujisawa H, Hasegawa M, Tachibana O, et al: Spinal epidural lipomatosis associated with pituitary macroprolactinoma. Acta Neurochir 144:213-214, 2002

6. Guegan Y, Fardoun R, Launois B, et al: Spinal cord compression by extradural fat after prolonged corticosteroid therapy. J Neurosurg 56:267-269, 1982

7. Iplikcioglu AC, Berkman MZ, Sengoz A: Idiopathic spinal epidural lipomatosis. Acta Neurochir 140:405-406, 1998

8. Kumar K, Nath RK, Nair CP, et al: Symptomatic epidural lipomatosis secondary to obesity. Case report. J Neurosurg 85: 348-350, 1996

9. Lisai P, Doria C, Crissantu L, et al: Cauda equina syndrome sec- ondary to idiopathic spinal epidural lipomatosis. Spine 26: 307-309, 2001

10. McCullen GM, Spurling GR, Webster JS: Epidural lipomatosis complicating lumbar steroid injections. J Spinal Disord 12: 526-529, 1999

11. Payer M, Van Schaeybroeck P, Reverdin A, et al: Idiopathic symptomatic epidural lipomatosis of the lumbar spine. Acta Neurochir 145:315-321, 2003

12. Perling LH, Laurent JP, Cheek WR: Epidural hibernoma as a complication of corticosteroid treatment. Case report. J Neurosurg 69:613-616, 1988

13. Robertson SC, Traynelis VC, Follett KA, et al: Idiopathic spinal epidural lipomatosis. Neurosurgery 41:68-75, 1997

14. Roy-Camille R, Mazel C, Husson JL, et al: Symptomatic spinal epidural lipomatosis induced by a long-term steroid treatment. Review of the literature and report of two additional cases. Spine 16:1365-1371, 1991

15. Sandberg DI, Lavyne MH: Symptomatic spinal epidural lipomatosis after local epidural corticosteroid injections: case report. Neurosurgery 45:162-165, 1999

16. Soloniuk DS, Pecoraro SR, Munschauer FE: Myelopathy secondary to spinal epidural lipomatosis. A case report. Spine 14: 119-122, 1989

17. Stambough JL, Cheeks ML, Keiper GL: Nonglucocorticoidinduced lumbar epidural lipomatosis: a case report and review of literature. J Spinal Disord 2:201-207, 1989

18. Stern JD, Quint DJ, Sweasey TA, et al: Spinal epidural lipomatosis: two new idiopathic cases and a review of the literature. J Spinal Disord 7:343-349, 1994

19. Tobler WD, Weil S: Epidural lipomatosis and renal transplantation. Surg Neurol 29:141-144, 1988

Manuscript received February 19, 2004.

Accepted in final form March 2, 2004.

Address reprint requests to: Meic H. Schmidt, M.D., Department of Neurosurgery, University of Utah School of Medicine, 30 North 1900 East, Suite 3B409, Salt Lake City, Utah 84132. email: meic.schmidt@hsc.utah.edu. 